Research Article

\title{
Point-Symmetric Extension-Based Interval Shannon-Cosine Spectral Method for Fractional PDEs
}

\author{
Ruyi Xing $\left(\mathbb{D},{ }^{1}\right.$ Yanqiao $\mathrm{Li}^{2}{ }^{2}$ Qing Wang, ${ }^{2}$ Yangyang $\mathrm{Wu},{ }^{2}$ and Shu-Li Mei $\mathbb{D}^{2}$ \\ ${ }^{1}$ Education Technology Center, Hebei University of Engineering, Handan 056038, China \\ ${ }^{2}$ College of Information and Electrical Engineering, China Agricultural University, Beijing 100083, China
}

Correspondence should be addressed to Shu-Li Mei; meishuli@163.com

Received 28 December 2019; Accepted 25 March 2020; Published 2 June 2020

Guest Editor: Fahd Jarad

Copyright (c) 2020 Ruyi Xing et al. This is an open access article distributed under the Creative Commons Attribution License, which permits unrestricted use, distribution, and reproduction in any medium, provided the original work is properly cited.

The approximation accuracy of the wavelet spectral method for the fractional PDEs is sensitive to the order of the fractional derivative and the boundary condition of the PDEs. In order to overcome the shortcoming, an interval Shannon-Cosine wavelet based on the point-symmetric extension is constructed, and the corresponding spectral method on the fractional PDEs is proposed. In the research, a power function of cosine function is introduced to modulate Shannon function, which takes full advantage of the waveform of the Shannon function to ensure that many excellent properties can be satisfied such as the partition of unity, smoothness, and compact support. And the interpolative property of Shannon wavelet is held at the same time. Then, based on the point-symmetric extension and the general variational theory, an interval Shannon-Cosine wavelet is constructed. It is proved that the first derivative of the approximated function with this interval wavelet function is continuous. At last, the wavelet spectral method for the fractional PDEs is given by means of the interval Shannon-Cosine wavelet. By means of it, the condition number of the discrete matrix can be suppressed effectively. Compared with Shannon and Shannon-Gabor wavelet quasi-spectral methods, the novel scheme has stronger applicability to the shockwave appeared in the solution besides the higher numerical accuracy and efficiency.

\section{Introduction}

In recent years, fractional calculus has been attracting more and more researchers in different fields of science and engineering and has been theoretically developed quickly over the last two decades [1-4]. It has been proved that the fractional-order differential equation models are more consistent with the biological phenomena [5] and hydrodynamics [6-8] than those of integer-orders. The Caputo and Riemann-Liouville fractional derivatives are the classical definition, and both of them have a kernel with singularity. To solve the problem of singular kernel, Caputo and Fabrizio proposed a derivative with fractional order based on the exponential function; their derivative in fact does not have singular kernel. In order to overcome the shortcoming of the nonlocal property of the exponential function, Ravichandran et al. [9-12] proposed two generalized fractional derivatives in Caputo and Riemann-Liouville sense [13, 14].

Despite a few special fraction PDEs having analytical solution [15], most of them should be solved by the numerical method. The solution of the fractional PDEs is sensitive to the iterative step, and so it is disabled to be solved by the traditional numerical method directly. Al-Mdallal has made outstanding contributions in this field, who has proposed many effective algorithms for solving the fractional PDEs, such as the fractional-order Legendre-collocation method [16] and fractional-Legendre spectral Galerkin method [17]. This inspired this work to try to construct a numerical method by means of the Shannon wavelet theory.

Sinc is a famous sampling function, but Shannon's reconstruction formula is rarely used in practice because of the slow decay of the Shannon function [18]. Taking the window functions to modulate the $\sin c$ function is the common method to improve its decay rate. Many windows have been proposed such as rectangular window, Bartlett window, Hanning window, Hamming window, and Blackman window.

In recent years, wavelet analysis theory has been developed to be a powerful tool to solve the fractional partial 
differential equations in recent years [19-21]. Shannon wavelets have been constructed based on the sinc function. A complex Shannon wavelet is defined by

$$
\psi(x)=\sqrt{f_{b}} \sin c\left(f_{b} x\right) e^{2 i \pi f_{c} x},
$$

where $f_{b}$ is the bandwidth and $f_{c}$ is the wavelet center frequency. Hoffman et al. [22] have presented the ShannonGabor wavelet as follows:

$$
\phi_{G}(x)=\frac{\sin (\pi x)}{\pi x} \exp \left(-\frac{x^{2}}{2 \sigma^{2}}\right), \quad \sigma>0
$$

where $\sigma$ is the width parameter (or called window size).

Both of the two Shannon-type wavelets are obtained by taking the Gaussian window to modulate the sinc function. The presence of the Gaussian window destroys the normative property possessed by the Shannon wavelet, that is,

$$
\widehat{\phi}_{G}(0)=\int_{-\infty}^{\infty} \phi_{G}(x) \mathrm{d} x=\operatorname{erf}\left(\frac{\pi \sigma}{\sqrt{2}}\right),
$$

where $\operatorname{erf}(z)=(2 / \sqrt{\pi}) \int_{0}^{z} e^{-t^{2}} \mathrm{~d} t$ is the error function. Obviously, $\widehat{\phi}_{\mathrm{G}}(0)$ is always less than unity except at the limit of $\sigma \longrightarrow \infty$. This is the reason why the windowed Shannon wavelet is not recommended. They fail to satisfy the partition of unity; this has the disturbing consequence that the reconstruction error will not vanish as the sampling step tends to zero.

Similar to Haar wavelet [23-25], B-Spline wavelet $[26,27]$, and Legendre wavelet $[28,29]$, Shannon wavelet $[30,31]$ possesses almost all the excellent numerical properties such as interpolative, relative sparse, and orthogonal properties besides the compact support property. Therefore, it is necessary to construct a novel window for sinc function, which can satisfy the partition of unity, so that it can be utilized to solve fractional PDEs efficiently [32]. In order to overcome the disadvantages in the Shannon-Gabor wavelet, a Shannon-Cosine wavelet is constructed by Mei et al. $[32,33]$, in which the waveform is used to meet the requirement of the partition of unity. Unfortunately, there are too many parameters in the Shannon-Cosine wavelet function to be identified, and this makes the expression to be very complex. In this paper, a simplified Shannon-Cosine wavelet function is proposed and the corresponding interval wavelet is constructed based on the point-symmetric extension [34, 35]. And then, the interval Shannon-Cosine wavelet is employed to construct a wavelet spectral method for the fractional PDEs.

\section{Simplified Shannon-Cosine Scaling Function}

As a basis function $\phi(x)=\sin c(x)$ defined in Hilbert space, it could form a basic approximation space $V$ as

$$
V(\phi)=\left\{s(x)=\sum_{k \in \mathbb{Z}} c(k) \phi(x-k), c \in l_{2}\right\} .
$$

This means that any function $s(x) \in V(\phi)$ is characterized by a sequence of coefficients $c(k)$. The coefficients $c(k)$ are the samples of the signal, and that $\phi(x)$ is a kind of weight function. Therefore, it should meet the requirements as follows:

(1) The coefficients sequence should be square-summable: $c \in l_{2}$.

(2) The family of functions $\left\{\phi_{k}=\phi(x-k)\right\}_{k \in \mathbb{Z}}$ should form a Riesz basis of $V(\phi)$. This ensures that the representation is stable and unambiguously defined. The Riesz basis requirement has an equivalent expression in the Fourier domain:

$$
A \leq \sum_{k \in \mathbb{Z}}|\widehat{\phi}(\omega+2 k \pi)|^{2} \leq B
$$

where $\widehat{\phi}(\omega)=\int \phi(x) e^{-j \omega x} \mathrm{~d} x$ is the Fourier transform of $\phi(x)$.

(3) $\phi(x)$ should satisfy the partition of unity condition $\int_{-\infty}^{\infty} \phi(x) \mathrm{d} x=1$.

Sinc is a famous sampling function [20] based on approximating the Dirac delta function as a band-limited function and is given by

$$
\phi(x)=\frac{\sin (\pi x)}{\pi x}
$$

In order to overcome the shortcomings of the ShannonGabor function, we introduce the parametric cosine function instead of the exponential function to modulate Shannon function as follows:

$$
S_{C}(x)=\frac{\sin (\pi x)}{\pi x} \cos ^{2 m}\left(\frac{\pi x}{N}\right)\left[\chi\left(x+\frac{N}{2}\right)-\chi\left(x-\frac{N}{2}\right)\right],
$$

where $N$ is a constant related to the support domain. $\chi(x)$ is the Heaviside function defined as follows:

$$
\chi(x)= \begin{cases}0, & x<0, \\ \text { undefined, } & x=0, \\ 1, & x>0 .\end{cases}
$$

This ensures that new modulate Shannon function (7) is a real compact support function, and the support domain is $[-N / 2, N / 2]$. The function $S_{C}(x)$ is named as ShannonCosine scaling function.

The comparison between the $\sin c$ and the ShannonCosine scaling function is shown in Figure 1.

It is easy to prove that $S_{C}(x)$ has the interpolative property; the coefficients $c(k)$ in equation (4) are the samples of the signal. Therefore, $c(k)$ obtained from any signal $s \in L_{2}$ satisfies the first requirement.

Based on the Parseval equation, it is easy to understand that any basis function with $\int_{-\infty}^{\infty} \phi(x) \mathrm{d} x=1$ meets the second requirement. This means that the third requirement puts the strongest constraint of the selection on an admissible generating function $\phi(x)$. It is well known that the $\sin c$ function satisfies the partition of unity condition, and this results in the Gaussian-windowed sinc function are not meeting this requirement. In the next section, we are going to prove that it can be satisfied by choosing the support domain parameter $N$. 


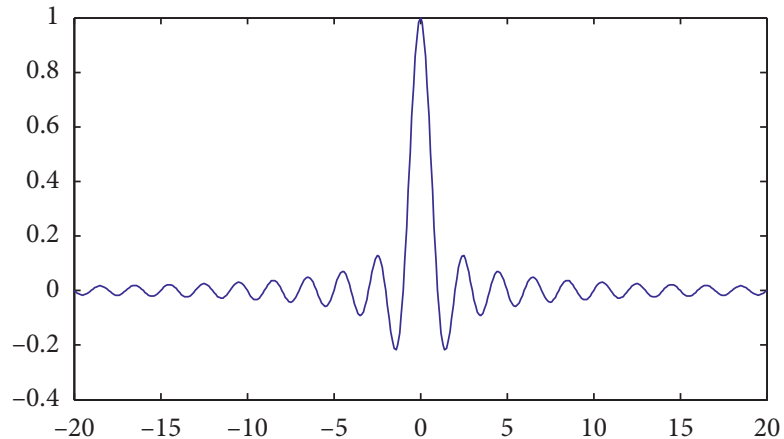

(a)

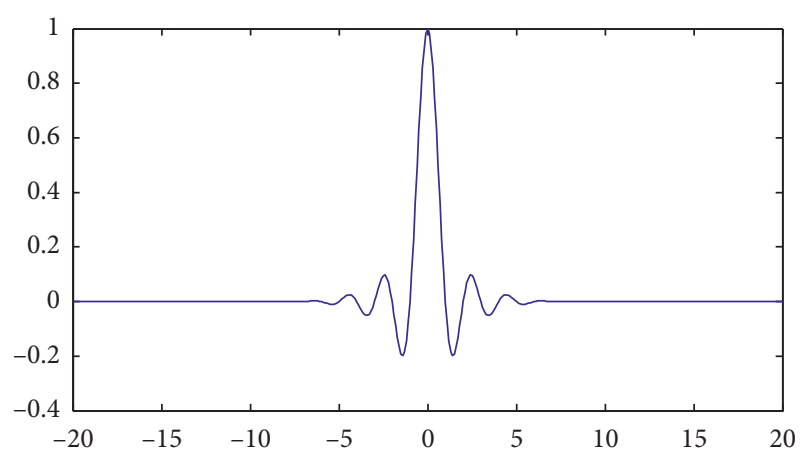

(b)

Figure 1: Comparison between Shannon and Shannon-Cosine scaling functions. (a) Shannon function. (b) Shannon-Cosine scaling function.

\section{Normalization and Choice of the Support Domain Parameter $\mathbf{N}$}

Shannon function has the waveform shown in Figure 2; its support domain is $(-\infty, \infty)$ and it meets the normalization condition $\int_{-\infty}^{\infty} \phi(x) \mathrm{d} x=1$. It is easy to notice that the integration value of Shannon function in domain $I_{1}$ is larger than 1 and smaller than 1 in domain $I_{2}$. This reminds us that the reasonable choice of the support domain can ensure that the Shannon-Cosine scaling function satisfied the partition of unity condition. Theorem 1 reveals the relation between the support domain parameter $N$ and the partition of unity condition.

Theorem 1. The Shannon-Cosine scaling function $(m=3)$ satisfies the normalization condition as follows:

$$
\int_{-\infty}^{\infty} S_{C}(x) \mathrm{d} x=1
$$

Proof. The Fourier transform of $S_{C}(x)$ is given by

$$
\begin{aligned}
\widehat{S}_{C}(\omega)= & \int_{-\infty}^{\infty} S_{C}(x) e^{-i \omega x} \mathrm{~d} x \\
= & \frac{1}{2 \pi} \sum_{n=0}^{3} a_{n}\left[\operatorname{Si}\left(\frac{1}{2} N \cdot(\pi+\omega)+n \pi\right)\right. \\
& +\operatorname{Si}\left(\frac{1}{2} N \cdot(\pi+\omega)-n \pi\right)+\operatorname{Si}\left(\frac{1}{2} N \cdot(\pi-\omega)+n \pi\right) \\
& \left.+\operatorname{Si}\left(\frac{1}{2} N \cdot(\pi-\omega)-n \pi\right)\right] .
\end{aligned}
$$

Let $m=3$. Substituting $\omega=0$ and equation (6) into equation (10), we obtain

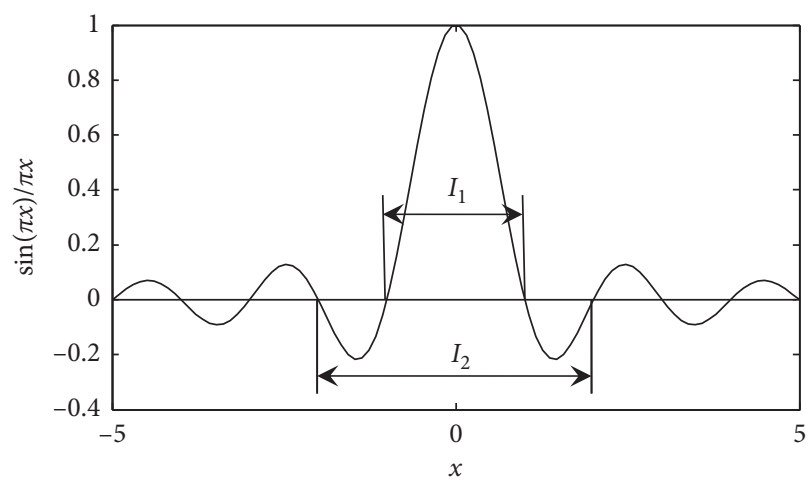

FIGURE 2: Shannon function.

$$
\begin{aligned}
\widehat{S}_{C}(0)= & \int_{-\infty}^{\infty} S_{C}(x) \mathrm{d} x \widehat{S}_{C}(0) \\
= & \frac{1}{\pi}\left[\frac{5}{16}\left(2 \operatorname{Si}\left(\frac{1}{2} N \pi\right)\right)+\frac{15}{32}\left(\operatorname{Si}\left(\frac{1}{2} N \pi+\pi\right)\right.\right. \\
& \left.+\operatorname{Si}\left(\frac{1}{2} N \pi-\pi\right)\right)+\frac{3}{16}\left(\operatorname{Si}\left(\frac{1}{2} N \pi+2 \pi\right)\right. \\
& \left.+\operatorname{Si}\left(\frac{1}{2} N \pi-2 \pi\right)\right)+\frac{1}{32}\left(\operatorname{Si}\left(\frac{1}{2} N \pi+3 \pi\right)\right. \\
& \left.\left.+\operatorname{Si}\left(\frac{1}{2} N \pi-3 \pi\right)\right)\right] .
\end{aligned}
$$

In fact, $\widehat{S}_{C}(0)$ can be viewed as a continuous function with respect to the real number " $N$," which is a parameter related to the length of the support domain. Let 


$$
\begin{aligned}
P(N)= & \widehat{S}_{C}(0) \\
= & \frac{1}{\pi}\left[\frac{5}{16}\left(2 \operatorname{Si}\left(\frac{1}{2} N \pi\right)\right)+\frac{15}{32}\left(\operatorname{Si}\left(\frac{1}{2} N \pi+\pi\right)\right.\right. \\
& \left.+\operatorname{Si}\left(\frac{1}{2} N \pi-\pi\right)\right)+\frac{3}{16}\left(\operatorname{Si}\left(\frac{1}{2} N \pi+2 \pi\right)\right. \\
& \left.+\operatorname{Si}\left(\frac{1}{2} N \pi-2 \pi\right)\right)+\frac{1}{32}\left(\operatorname{Si}\left(\frac{1}{2} N \pi+3 \pi\right)\right. \\
& \left.\left.+S i\left(\frac{1}{2} N \pi-3 \pi\right)\right)\right] .
\end{aligned}
$$

Based on the definition of $\operatorname{Si}(x)$, we know that $P(N)$ is an oscillator function around the constant 1 , that is,

$$
\begin{aligned}
& P(N)\left\{\begin{array}{l}
>1, \quad \frac{N}{2}=2 n, n \geq 2, \\
<1, \quad \frac{N}{2}=2 n+1, n \geq 2,
\end{array}\right. \\
& P^{\prime}(N)=\frac{\mathrm{d} P(N)}{\mathrm{d} N} \\
& \quad=-\frac{1440 \sin (N \pi / 2)}{\pi N\left(N^{2}-4\right)\left(N^{2}-16\right)\left(N^{2}-36\right)} .
\end{aligned}
$$

Obviously,

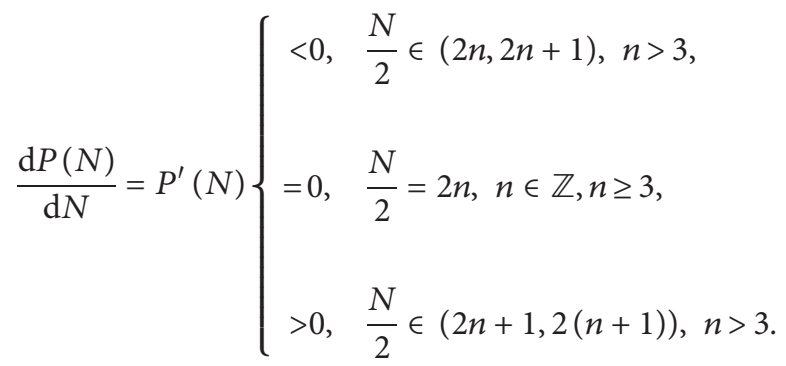

That is, $N=4 n, n \in \mathbb{Z}, n \geq 3$, is in correspondence with the extreme point of the function $P(N)$, which is a
TABLE 1: Solution of $P(N)=1$.

\begin{tabular}{lll}
\hline 7.437330346546332 & 9.323969185188616 & 11.261199765911442 \\
13.219709116325248 & 15.189881678437814 & 17.167295939754695 \\
19.149560460820794 & 21.135248098522425 & 23.123447719961405 \\
25.113547757267952 & 27.105121575295925 & 29.097861737012863 \\
31.091541469097137 & 33.085988700389862 & 35.081073641777039 \\
37.076687097549438 & 39.072752714157104 & 41.069202184677124 \\
43.065983057022095 & 45.063048839569092 & 47.060366630554199 \\
49.057900428771973 & 51.055631637573242 & 53.053528785705566 \\
55.051587104797363 & 57.049772262573242 & 59.048089981079102 \\
61.046504974365234 & 63.045036315917969 & 65.043693542480469 \\
67.042350769042969 & 69.041099548339844 & \\
\hline
\end{tabular}

monotone function on the intervals $(2 n, 2 n+1)$ and $(2 n+1$, $2 n+2)$. Based on the mean value theorem, there must exist unique points $N / 2 \in(2 n, 2 n+1)$ and $N / 2 \in(2 n, 2 n+2)$ so as to $P(N)=1$, i.e.,

$$
P(N)=\widehat{S}_{C}(0)=\int_{-\infty}^{\infty} S_{C}(x) \mathrm{d} x=1 .
$$

This completes the proof.

By means of the interval bisection method, it is easy to obtain the value of $N$ which is in correspondence with $P(N)=1$ as in Table 1 .

It is easy to prove that the simplified Shannon-Cosine wavelet is equivalent to the Shannon-Cosine wavelet proposed in [36] using the parameter $m \leq 3$, but only the parameter $N$ should be chosen in applications. So, it is convenient for solving the fractional derivative PDEs.

\section{Point-Symmetric Interval Wavelet}

In order to eliminate the boundary effect introduced by the wavelet transform, the point-symmetric extension and the general variational theory are employed to construct the interval wavelet. Compared to other extension methods such as the zero extension, symmetric extension, and periodic extension, the point-symmetric extension can ensure that the function is smooth at the endpoint.

Theorem 2. If the continuous function $f(x)$ is symmetric on point $\left(x_{0}, f\left(x_{0}\right)\right)$, then the first-order derivative of the function $f(x)$ is continuous at this point.

Proof. As the continuous function $f(x)$ is symmetric on point $\left(x_{0}, f\left(x_{0}\right)\right)$, let $\Delta x>0$; we have 


$$
\begin{aligned}
f\left(x_{0}-\Delta x\right) & =2 f\left(x_{0}\right)-f\left(x_{0}+\Delta x\right), \\
\lim _{x_{0}-\Delta x \longrightarrow x_{0}} \frac{f\left(x_{0}-\Delta x\right)-f\left(x_{0}\right)}{x_{0}-\Delta x-x_{0}} & =\lim _{x_{0}-\Delta x \longrightarrow x_{0}} \frac{f\left(x_{0}-\Delta x\right)-f\left(x_{0}\right)}{-\Delta x} \\
& =\lim _{x_{0}-\Delta x \longrightarrow x_{0}} \frac{2 f\left(x_{0}\right)-f\left(x_{0}+\Delta x\right)-f\left(x_{0}\right)}{-\Delta x} \\
& =\lim _{\Delta x \longrightarrow 0} \frac{f\left(x_{0}+\Delta x\right)-f\left(x_{0}\right)}{\Delta x}, \\
\lim _{x_{0}+\Delta x \longrightarrow x_{0}} \frac{f\left(x_{0}+\Delta x\right)-f\left(x_{0}\right)}{x_{0}+\Delta x-x_{0}} & =\lim _{x_{0}+\Delta x \rightarrow x_{0}} \frac{f\left(x_{0}+\Delta x\right)-f\left(x_{0}\right)}{\Delta x} \\
& =\lim _{\Delta x \longrightarrow 0} \frac{f\left(x_{0}+\Delta x\right)-f\left(x_{0}\right)}{\Delta x} .
\end{aligned}
$$

Therefore, we have

$$
\lim _{x_{0}-\Delta x \rightarrow x_{0}} \frac{f\left(x_{0}-\Delta x\right)-f\left(x_{0}\right)}{x_{0}-\Delta x-x_{0}}=\lim _{x_{0}+\Delta x \longrightarrow x_{0}} \frac{f\left(x_{0}+\Delta x\right)-f\left(x_{0}\right)}{x_{0}+\Delta x-x_{0}} .
$$

This illustrates that the first derivative of the function $f(x)$ continues to reach the point $\left(x_{0}, f\left(x_{0}\right)\right)$. This completes the proof.

For convenience, the values of $f(x)$ at $x_{-n}, x_{-n+1}, \ldots, x_{0}$, $x_{1}, \ldots, x_{2^{j}+1}$ are denoted as $f_{-n}, f_{-n+1}, \ldots, f_{0}, f_{1}, \ldots, f_{2^{j+1}}$. Based on the general variational theory, we can deduce the format of the interval wavelet function as follows:

(1) As $x=-n,-n+1, \ldots,-1$,

$$
f_{-1}=\left(2 f_{0}-f_{1}\right) \cos ^{2}(\pi / 2 n)
$$

$$
f_{-2}=\left(2 f_{0}-f_{2}\right) \cos ^{2}(2 \pi / 2 n)
$$$$
\ddot{f}_{-k}=\left(2 f_{0}-f_{k}\right) \cos ^{2}(k \pi / 2 n)
$$$$
\ddot{f}_{-N}=\left(2 f_{0}-f_{N}\right) \cos ^{2}(\pi / 2)
$$

(2) As $x=2^{j}+1,2^{j}+2, \ldots, 2^{j}+n$,

$$
\begin{aligned}
& f_{2^{j+1}}=\left(2 f_{2^{j}}-f_{2^{j_{-}}}\right) \cos ^{2}(\pi / 2 n) \\
& f_{2^{j+2}}=\left(2 f_{2^{j}}-f_{2^{j}-2}\right) \cos ^{2}(2 \pi / 2 n) \\
& \ldots_{2^{j+k}}=\left(2 f_{2^{j}}-f_{2^{j-k}-k}\right) \cos ^{2}(k \pi / 2 n) \\
& \ldots \\
& f_{2^{j+n}}=\left(2 f_{2^{j}}-f_{2^{j-n}}\right) \cos ^{2}(n \pi / 2 n)
\end{aligned}
$$

And so, the function $f(x)$ can be expressed as

$$
\begin{aligned}
f(x)= & \sum_{k=-n}^{2^{j}+n} \phi\left(2^{j} x-k\right) f_{k}^{j} \\
= & \sum_{k=-n}^{-1} \phi\left(2^{j} x-k\right)\left(2 f_{0}-f_{-k}^{j}\right) \cos ^{2}\left(\frac{k \pi}{2 n}\right)+\sum_{k=0}^{2^{j}} \phi\left(2^{j} x-k\right) f_{k}^{j}+\sum_{k=2^{j}+1}^{2^{j}+n} \phi\left(2^{j} x-k\right)\left(2 f_{2^{j}}-f_{2^{j}-k}\right) \cos ^{2}\left(\frac{\left(k-2^{j}\right) \pi}{2 n}\right) \\
= & -\sum_{k=-n}^{-1} \phi\left(2^{j} x-k\right) \cos ^{2}\left(\frac{k \pi}{2 n}\right) f_{-k}^{j}+2 \sum_{k=-n}^{-1} \phi\left(2^{j} x-k\right) \cos ^{2}\left(\frac{k \pi}{2 n}\right) f_{0}+\sum_{k=0}^{2^{j}} \phi\left(2^{j} x-k\right) f_{k}^{j} \\
& +2 \sum_{k=2^{j}+1}^{2^{j}+n} \phi\left(2^{j} x-k\right) \cos ^{2}\left(\frac{\left(k-2^{j}\right) \pi}{2 n}\right) f_{2^{j}}-\sum_{k=2^{j}+1}^{2^{j}+n} \phi\left(2^{j} x-k\right) \cos ^{2}\left(\frac{\left(k-2^{j}\right) \pi}{2 n}\right) f_{2^{j+1}-k} \\
= & -\sum_{k=1}^{n} \phi\left(2^{j} x+k\right) \cos ^{2}\left(\frac{k \pi}{2 n}\right) f_{k}^{j}+2 \sum_{k=1}^{n} \phi\left(2^{j} x+k\right) \cos ^{2}\left(\frac{k \pi}{2 n}\right) f_{0}+\sum_{k=0}^{2^{j}} \phi\left(2^{j} x-k\right) f_{k}^{j} \\
& +2 \sum_{k=-2^{j}-n}^{-2^{j}-1} \phi\left(2^{j} x+k\right) \cos ^{2}\left(\frac{\left(k+2^{j}\right) \pi}{2 n}\right) f_{2^{j}}-\sum_{k=2^{j}+1}^{2^{j}+n} \phi\left(2^{j} x-k\right) \cos ^{2}\left(\frac{\left(k-2^{j}\right) \pi}{2 n}\right) f_{2^{j+1}-k} .
\end{aligned}
$$


Let $m=2^{j+1}-k$; we have

$$
\begin{aligned}
f(x)= & -\sum_{k=1}^{n} \phi\left(2^{j} x+k\right) \cos ^{2}\left(\frac{k \pi}{2 n}\right) f_{k}^{j}+2 \sum_{k=-n}^{-1} \phi\left(2^{j} x-k\right) \cos ^{2}\left(\frac{k \pi}{2 n}\right) f_{0}+\sum_{k=0}^{2^{j}} \phi\left(2^{j} x-k\right) f_{k}^{j} \\
& +2 \sum_{k=2^{j}+1}^{2^{j}+n} \phi\left(2^{j} x-k\right) \cos ^{2}\left(\frac{\left(2^{j}-k\right) \pi}{2 n}\right) f_{2^{j}}-\sum_{m=2^{j}-n}^{2^{j-1}} \phi\left(2^{j} x-2^{j+1}+m\right) \cos ^{2}\left(\frac{\left(2^{j}-m\right) \pi}{2 n}\right) f_{m},
\end{aligned}
$$

where

So, we have

$$
\begin{aligned}
& \sum_{k=0}^{2^{j}} \phi\left(2^{j} x-k\right) f_{k}^{j}=\phi\left(2^{j} x\right) f_{0}^{j}+\sum_{k=1}^{n} \phi\left(2^{j} x-k\right) f_{k}^{j} \\
& \quad+\sum_{k=n+1}^{2^{j}-n-1} \phi\left(2^{j} x-k\right) f_{k}^{j}+\sum_{k=2^{j}-n}^{2^{j}-1} \phi\left(2^{j} x-k\right) f_{k}^{j}+\phi\left(2^{j} x-2^{j}\right) f_{2^{j}}^{j} .
\end{aligned}
$$

$$
\begin{aligned}
f(x)= & \left(\phi\left(2^{j} x\right)+2 \sum_{k=-n}^{-1} \phi\left(2^{j} x-k\right) \cos ^{2}\left(\frac{k \pi}{2 n}\right)\right) f_{0}^{j}+\sum_{k=1}^{n}\left(\phi\left(2^{j} x-k\right)-\phi\left(2^{j} x+k\right) \cos ^{2}\left(\frac{k \pi}{2 n}\right)\right) f_{k}^{j} \\
& +\sum_{k=N+1}^{2^{j}-N-1} \phi\left(2^{j} x-k\right) f_{k}^{j}+\sum_{k=2^{j}-N}^{2^{j}-1}\left(\phi\left(2^{j} x-k\right)-\phi\left(2^{j} x-2^{j+1}+k\right) \cos ^{2}\left(\frac{\left(2^{j}-k\right) \pi}{2 n}\right)\right) f_{k}^{j} \\
& +\left(\phi\left(2^{j} x-2^{j}\right)+2 \sum_{k=2^{j}+1}^{2^{j}+N} \phi\left(2^{j} x-k\right) \cos ^{2}\left(\frac{\left(2^{j}-k\right) \pi}{2 n}\right)\right) f_{2^{j}} .
\end{aligned}
$$

Therefore, the interval interpolative wavelet function can be expressed as

$$
\begin{aligned}
& w\left(2^{j} \mathbf{x}-\mathbf{k}\right)=w_{j, k} \\
& = \begin{cases}\phi\left(2^{j} \mathbf{x}-\mathbf{k}\right)+2 \sum_{\mathbf{k}=1}^{\mathbf{P}} \phi\left(2^{j} \mathbf{x}+\mathbf{k}\right) \cos ^{2}\left(\frac{k \pi}{2 P}\right), & \mathbf{k}=0, \\
\phi\left(2^{j} \mathbf{x}-\mathbf{k}\right)-\phi\left(2^{j} \mathbf{x}+\mathbf{k}\right) \cos ^{2}\left(\frac{k \pi}{2 P}\right), & \mathbf{k}=1,2, \ldots, P, \\
\phi\left(2^{j} \mathbf{x}-\mathbf{k}\right), & \mathbf{k}=\mathbf{N}+1, \ldots, 2^{j}-P-1, \\
\phi\left(2^{j} \mathbf{x}-\mathbf{k}\right)-\phi\left(2^{j} \mathbf{x}-2^{j+1}+\mathbf{k}\right) \cos ^{2}\left(\frac{\left(2^{j}-k\right) \pi}{2 P}\right), & \mathbf{k}=2^{j}-P, \ldots, 2^{j}-1, \\
\phi\left(2^{j} \mathbf{x}-2^{\mathbf{j}}\right)+2 \sum_{\mathbf{k}=2^{j}+1}^{2^{j}+\mathbf{N}} \phi\left(2^{j} \mathbf{x}-\mathbf{k}\right) \cos ^{2}\left(\frac{\left(2^{j}-k\right) \pi}{2 P}\right), & \mathbf{k}=2^{j} .\end{cases}
\end{aligned}
$$


According to theory 2, it is easy to understand that equation (22) is able to reduce the boundary effect efficiently. In theory, the proposed interval wavelet can prevent the steep shock wave appearing near the boundary, which can introduce the large condition number of the discrete matrix and result in the decrease of the numerical precision.

\section{Application in Solving Fractional PDEs}

5.1. Interval Wavelet Spectral Method for Fractional Fokker-Planck Equation. The fractional Fokker-Planck equation is a typical fractional PDE, which is often used to describe a subdiffusive behavior of a particle under the combined influence of external nonlinear force field and a Boltzmann thermal heat bath. In the presence of an externalforce field $F(\mathbf{x})=-v^{\prime}(x)$, the evolution of a test particle is usually described in terms of the Fokker-Planck equation (FPE):

$$
\begin{array}{r}
\frac{\partial u(x, t)}{\partial t}={ }_{0} D_{t}^{1-\alpha}\left[\frac{\partial}{\partial x} \frac{\nu^{\prime}(x)}{m \eta_{\alpha}}+K_{\alpha} \frac{\partial^{2}}{\partial x^{2}}\right] u(x, t), \\
a \leq x \leq b, 0 \leq t \leq T,
\end{array}
$$

which defines the probability $u(x, t)$ of finding the particle at a certain position $x$ at a given time $t . m$ denotes the mass of the diffusing particle, $K_{\alpha}>0$ denotes the generalized diffusion coefficient with dimension $\left[K_{\alpha}\right]=\mathrm{cm}^{2} \mathrm{sec}^{-\alpha}$, and $\eta_{\alpha}$ is the generalized friction coefficient with dimension $\left[\eta_{\alpha}\right]=\sec ^{\alpha-2}$. The corresponding initial condition is

$$
u(x, 0)=\varphi(x), \quad a \leq x \leq b,
$$

and the boundary conditions are

$$
\begin{aligned}
u(a, t) & =p_{1}(t), \\
u(b, t) & =p_{2}(t), \\
& 0<t \leq T .
\end{aligned}
$$

Equation (2) uses the Riemann-Liouville fractional derivative of order $1-\alpha$, defined by

$$
{ }_{0} D_{t}^{1-\alpha} u(x, t)=\frac{1}{\Gamma(\alpha)} \frac{\partial}{\partial t} \int_{0}^{1} \frac{u(x, \eta)}{(t-\eta)^{1-\alpha}} d \eta, \quad 0 \leq \alpha<1,
$$

where $\Gamma(\alpha)$ is the gamma function.

According to the properties of the Riemann-Liouville fractional derivative, it is easy to know that, if $(x, t) \in C_{x, t}^{2,1}([a, b] \times[0, T])$, equation (2) can be rewritten as follows:

$$
\begin{array}{r}
D_{t}^{\alpha} u(x, t)-\frac{u(x, 0) t^{-\alpha}}{\Gamma(1-\alpha)}=\left[\frac{\partial}{\partial x} \frac{v^{\prime}(x)}{m \eta_{\alpha}}+K_{\alpha} \frac{\partial^{2}}{\partial x^{2}}\right] u(x, t), \\
a \leq x \leq b, 0 \leq t \leq T .
\end{array}
$$

According to the wavelet spectral method, the fractional Fokker-Planck equation can be approximately represented as

$$
\begin{gathered}
\tau^{-\alpha}\left[u_{j}\left(x_{i}, t_{n}\right)+\sum_{k=1}^{n-1} g_{k} u_{j}\left(x_{i}, t_{n-k}\right)-\sum_{k=0}^{n-1} g_{k} u_{j}\left(x_{i}, t_{0}\right)\right] \\
=f^{\prime}\left(x_{i}\right) u_{j}\left(x_{i}, t_{n}\right)+\sum_{m=0}^{2^{j}} u_{j}\left(x_{m}, t_{n}\right)\left[w^{\prime}\left(x_{i}-x_{m}\right)\right. \\
\left.+K_{\alpha} w^{\prime \prime}\left(x_{i}-x_{m}\right)\right],
\end{gathered}
$$

where $i=0,1,2, \ldots, 2^{j}$. Let

$$
\begin{aligned}
V_{j}^{n} & =\left(u_{j}\left(x_{0}, t_{n}\right), u_{j}\left(x_{1}, t_{n}\right), \ldots, u_{j}\left(x_{2^{j}}, t_{n}\right)\right)^{T}, \\
F & =\operatorname{diag}\left(f^{\prime}\left(x_{0}\right), f^{\prime}\left(x_{1}\right), \ldots, f^{\prime}\left(x_{2^{j}}\right)\right), \\
W_{1} & =\left[\begin{array}{cccc}
w^{\prime}\left(x_{0}-x_{0}\right) & w^{\prime}\left(x_{0}-x_{1}\right) & \cdots & w^{\prime}\left(x_{0}-x_{2^{j}}\right) \\
w^{\prime}\left(x_{1}-x_{0}\right) & w^{\prime}\left(x_{1}-x_{1}\right) & \cdots & w^{\prime}\left(x_{1}-x_{2^{j}}\right) \\
\vdots & \vdots & \ddots & \vdots \\
w^{\prime}\left(x_{2^{j}}-x_{0}\right) & w^{\prime}\left(x_{2^{j}}-x_{1}\right) & \cdots & w^{\prime}\left(x_{2^{j}}-x_{2^{j}}\right)
\end{array}\right], \\
W_{2} & =\left[\begin{array}{cccc}
w^{\prime \prime}\left(x_{0}-x_{0}\right) & w^{\prime \prime}\left(x_{0}-x_{1}\right) & \cdots & w^{\prime \prime}\left(x_{0}-x_{2^{j}}\right) \\
w^{\prime \prime}\left(x_{1}-x_{0}\right) & w^{\prime \prime}\left(x_{1}-x_{1}\right) & \cdots & w^{\prime \prime}\left(x_{1}-x_{2^{j}}\right) \\
\vdots & \vdots & \ddots & \vdots \\
w^{\prime \prime}\left(x_{2^{j}}-x_{0}\right) & w^{\prime \prime}\left(x_{2^{j}}-x_{1}\right) & \cdots & w^{\prime \prime}\left(x_{2^{j}}-x_{2^{j}}\right)
\end{array}\right] .
\end{aligned}
$$
format:

Then, the system of (28) can be expressed in the matrix

$$
\left(W_{1}+K_{\alpha} W_{2}+F-\tau^{-\alpha} I\right) V_{j}^{n}=\sum_{k=1}^{n-1} g_{k} V_{j}^{n-k}-\sum_{k=0}^{n-1} g_{k} V_{j}^{0} .
$$

5.2. Numerical Experiments. In this section, a simple Fokker-Planck equation with the exact analytical solution is taken as the example to illustrate the effectiveness. Consider the Fokker-Planck equation as follows:

$$
\frac{\partial u(x, t)}{\partial t}={ }_{0} D_{t}^{1-\alpha}\left[\frac{\partial}{\partial x}(-1)+\frac{\partial^{2}}{\partial x^{2}}\right] u(x, t), \quad 0 \leq x \leq 1, t>0 .
$$

With the initial condition

$$
u(x, 0)=x(1-x), \quad 0 \leq x \leq 1,
$$

the boundary conditions are

$$
\begin{aligned}
& u(0, t)=-\frac{3 t^{\alpha}}{\Gamma(1+\alpha)}-\frac{2 t^{2 \alpha}}{\Gamma(1+2 \alpha)}, \quad t>0, \\
& u(1, t)=-\frac{t^{\alpha}}{\Gamma(1+\alpha)}-\frac{2 t^{2 \alpha}}{\Gamma(1+2 \alpha)}, \quad t>0 .
\end{aligned}
$$

The exact analytic solution is

$$
u(x, t)=x(1-x)+(2 x-3) \frac{t^{\alpha}}{\Gamma(1+\alpha)}-\frac{2 t^{2 \alpha}}{\Gamma(1+2 \alpha)} .
$$


TABLE 2: Influence of $\alpha$ on the numerical precision $(t=0.0001, T=0.1)$.

\begin{tabular}{|c|c|c|c|c|c|c|c|}
\hline \multirow[b]{2}{*}{$j$} & \multirow[b]{2}{*}{$\alpha$} & \multicolumn{3}{|c|}{$e_{1}$} & \multicolumn{3}{|c|}{$e_{2}$} \\
\hline & & $\begin{array}{l}\text { Point-symmetric } \\
\text { interval WPM }\end{array}$ & $\begin{array}{l}\text { Lagrange interval } \\
\text { WPM }(L=2)\end{array}$ & $\begin{array}{c}\text { Dynamic interval } \\
\text { WPM }\end{array}$ & $\begin{array}{l}\text { Point-symmetric } \\
\text { interval WPM }\end{array}$ & $\begin{array}{l}\text { Lagrange interval } \\
\text { WPM }(L=2)\end{array}$ & $\begin{array}{c}\text { Dynamic interval } \\
\text { WPM }\end{array}$ \\
\hline \multirow{4}{*}{6} & 0.8 & 0.0012 & $6.2776 \times 10^{-6}$ & $3.5310 \times 10^{-4}$ & $9.5851 \times 10^{-4}$ & $4.9985 \times 10^{-6}$ & $8.3847 \times 10^{-5}$ \\
\hline & 0.6 & 0.0079 & $9.6280 \times 10^{179}$ & 0.0096 & 0.0066 & inf & 0.0076 \\
\hline & 0.4 & 0.0509 & 0 & 0.0588 & 0.0433 & $\mathrm{NaN}$ & 0.0499 \\
\hline & 0.2 & 0.3488 & 0 & 0.3962 & 0.2909 & $\mathrm{NaN}$ & 0.3284 \\
\hline \multirow{4}{*}{7} & 0.8 & 0.0012 & $1.9290 \times 10^{155}$ & 0.0014 & $9.9232 \times 10^{-4}$ & inf $^{4}$ & 0.0012 \\
\hline & 0.6 & 0.008 & 0 & 0.0092 & 0.0067 & $\mathrm{NaN}$ & 0.0077 \\
\hline & 0.4 & 0.0511 & 0 & 0.0586 & 0.0437 & $\mathrm{NaN}$ & 0.0502 \\
\hline & 0.2 & 0.3503 & 0 & 0.3932 & 0.2936 & $\mathrm{NaN}$ & 0.3282 \\
\hline
\end{tabular}

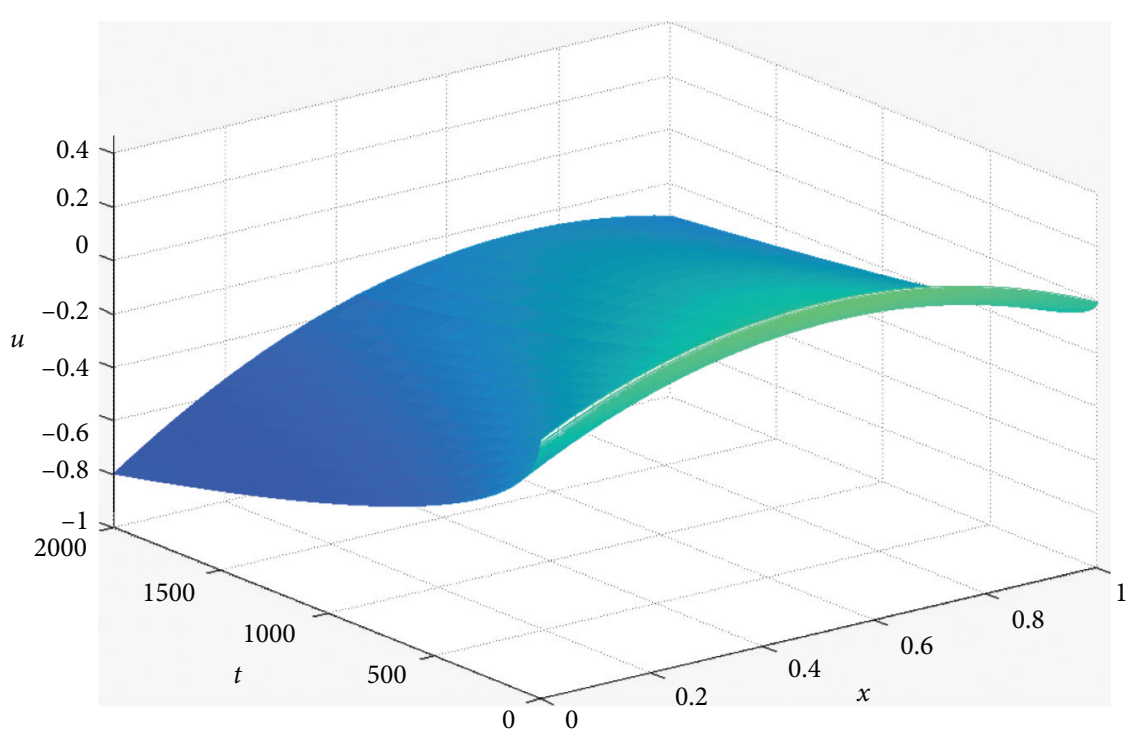

FIgURE 3: Trend of the solution with $t$.

All the comparisons in this section are made qualitatively by comparing the calculation precision in the same time step and space mesh grid size. The first measure of error $e_{1}$ is given by

$$
e_{1}=\left\|V_{j}^{n}-V_{\text {exact }}^{n}\right\|_{\infty} .
$$

It provides a measure of the accuracy of the solution near the boundary. The second measure of error $e_{2}$ is given by

$$
e_{2}=\sqrt{\frac{1}{2^{j}+1} \sum_{i=0}^{2^{j}}\left(u\left(x_{i}\right)-u_{\text {exact }}\left(x_{i}\right)\right)^{2}} \text {. }
$$

It provides a general measure of the accuracy of the solution over the main body of the distribution and was often used to investigate the accuracy of the FEM.

In [24], the dynamic interval wavelet spectral method (WPM) is employed to solve the fractional Fokker-Planck equation, in which the Lagrange interpolation-based interval wavelet spectral method is taken to compare with their method. Compared to these two methods, as shown in Table 2, the proposed method is more robust and insensitive to the parameters $j$ and $\alpha$. With the increase in the discrete point amount and decrease in the parameter $\alpha$, the numerical precision of the proposed method is better than of the dynamic interval WPM.

It should be noticed that the numerical precision of the dynamic interval WPM is better than the proposed method as $j=6$ and $\alpha=0.8$. The amount of the discrete points is $2^{j}$. This denotes that the numerical precision obtained by fewer discrete points is better than that obtained by more points. This is obviously unreasonable.

In fact, the dynamic interval wavelet function is constructed based on the Lagrange interpolation. The condition number of the discrete matrix is becoming very large with the increase in the Lagrange polynomial degree [36]. This is the primary reason why the numerical precision with fewer discrete points is better than more points by the dynamic interval WPM. Theorem 2 illustrates that the point-symmetric interval wavelet method can overcome this shortcoming of the dynamic interval wavelet as it does not have the steep wave close to the boundary point introduced by the Lagrange interpolation.

The trend of the solution with the parameter " $t$ " obtained by the proposed method is shown in Figure 3. This illustrates 
that the point-symmetric interval WPM is a robust method for the fractional PDEs.

\section{Conclusions}

By means of the waveform of the sinc function, a family of simplified Shannon-Cosine scaling functions is presented, which can be utilized to construct the wavelet spectral method for solving the fractional PDEs, combining with the point-symmetric extension, the solutions of which are permitted to have different smoothness. Compared with the $\sin c$ and Shannon-Gabor functions, the Shannon-Cosine scaling functions possess almost all the excellent numerical properties such as the compact support, interpolation, and derivability. The point-symmetric extension-based interval wavelet is a basis with robust properties, which can prevent the increase in the condition number introduced by the Lagrange interpolation. Besides, the proposed interval wavelet need not choose the smoothness of the approximation function near the boundary points, and this is helpful to improve the efficiency of the algorithm. Based on the choice scheme of the parameters appeared in the simplified Shannon-Cosine wavelet function, the adaptability of the spectral method to the smoothness of the solution can be improved greatly at even fewer collocation points. Compared with the Shannon-Cosine wavelets, there are fewer parameters in the simplified wavelet function, and this brings us a lot of conveniences in solving PDEs.

\section{Data Availability}

The MATLAB source code supporting the findings of this study has been deposited in the GitHub repository (https:// github.com/meishuli/meishuli/tree/Matlab-source-code).

\section{Conflicts of Interest}

The authors declare that they have no conflicts of interest.

\section{Acknowledgments}

The authors would like to thank their colleagues for all their help and support during this work. This work was supported by the National Natural Science Foundation of China (Grant No. 61871380) and Beijing Natural Science Foundation (Grant No. 4172034).

\section{References}

[1] F. A. Rihan, Q. M. Al-Mdallal, H. J. AlSakaji, and A. Hashish, "A fractional-order epidemic model with time-delay and nonlinear incidence rate," Chaos, Solitons \& Fractals, vol. 126, pp. 97-105, 2019.

[2] Q. Al-Mdallal, A. K. Ali, and I. Khan, "Analytical solutions of fractional walter's b fluid with applications," Complexity, vol. 2018, Article ID 8131329, 10 pages, 2018.

[3] Q. M. Al-Mdallal, T. Abdeljawad, and M. A. Hajji, "Theoretical and numerical results for fractional difference and differential equations," Discrete Dynamics in Nature And Society, vol. 2017, Article ID 2543452, 2 pages, 2017.
[4] S. Kirmani, N. Binti, and M. B. Riaz, "Shape preserving fractional order KNR C 1 cubic spline," European Physical Journal Plus, vol. 134, no. 7, pp. 1-8, 2019.

[5] F. A. Rihan, A. Hashish, F. Al-Maskari et al., "Dynamics of tumor-immune system with fractional-order," Journal of Tumor Research, vol. 2, no. 1, pp. 109-115, 2016.

[6] M. A. Imran, M. Aleem, M. B. Riaz, R. Ali, and I. Khan, "A comprehensive report on convective flow of fractional (ABC) and (CF) MHD viscous fluid subject to generalized boundary conditions," Chaos, Solitons \& Fractals, vol. 118, pp. 274-289, 2019.

[7] M. A. Imran, M. B. Riaz, N. A. Shah, and A. A. Zafar, "Boundary layer flow of MHD generalized Maxwell fluid over an exponentially accelerated infinite vertical surface with slip and Newtonian heating at the boundary," Results in Physics, vol. 8, pp. 1061-1067, 2018.

[8] N. A. Asif, Z. Hammouch, M. B. Riaz, and H. Bulut, "Analytical solution of a Maxwell fluid with slip effects in view of the Caputo-Fabrizio derivative?" European Physical Journal Plus, vol. 133, no. 7, pp. 272-279, 2018.

[9] C. Ravichandran, N. Valliammal, and J. J. Nieto, "New results on exact controllability of a class of fractional neutral integrodifferential systems with state-dependent delay in Banach spaces," Journal of the Franklin Institute, vol. 356, no. 3, pp. 1535-1565, 2019.

[10] C. Ravichandran, K. Logeswari, and F. Jarad, "New results on existence in the framework of Atangana-Baleanu derivative for fractional integro-differential equations," Chaos, Solitons \& Fractals, vol. 125, pp. 194-200, 2019.

[11] K. Logeswari and C. Ravichandran, "A new exploration on existence of fractional neutral integro-differential equations in the concept of Atangana-Baleanu derivative," Physica A: Statistical Mechanics and Its Applications, vol. 544, Article ID 123454, 2020.

[12] R. Subashini, C. Ravichandran, K. Jothimani, and H. M. Baskonus, "Existence results of Hilfer integro-differential equations with fractional order," Discrete \& Continuous Dynamical Systems - S, vol. 13, no. 3, pp. 911-923, 2020.

[13] N. Valliammal, C. Ravichandran, and Z. Hammouch, "A new investigation on fractional-ordered neutral differential systems with state-dependent delay," International Journal of Nonlinear Sciences and Numerical Simulation, vol. 20, no. 7-8, pp. 803-809, 2019.

[14] O. J. J. Algahtani, "Comparing the Atangana-Baleanu and Caputo-Fabrizio derivative with fractional order: allen Cahn model," Chaos, Solitons \& Fractals, vol. 89, pp. 552-559, 2016.

[15] M. B. Riaz, M. A. Imran, and K. Shabbir, "Analytic solutions of Oldroyd-B fluid with fractional derivatives in a circular duct that applies a constant couple," Alexandria Engineering Journal, vol. 55, no. 4, pp. 3267-3275, 2016.

[16] Q. M. Al-Mdallal and A. S. Abu Omer, "Fractional-order Legendre-collocation method for solving fractional initial value problems," Applied Mathematics and Computation, vol. 321, pp. 74-84, 2018.

[17] Q. M. Al-Mdallal and M. Qasem, "On fractional-Legendre spectral Galerkin method for fractional Sturm-Liouville problems," Chaos, Solitons \& Fractals, vol. 116, pp. 261-267, 2018.

[18] M. Unser, "Sampling-50 years after shannon," Proceedings of The IEEE, vol. 88, no. 4, pp. 569-587, 2000.

[19] H. Q. Kafri and S. A. Khuri, "Bratu's problem: a novel approach using fixed-point iterations and Green's functions," Computer Physics Communications, vol. 198, pp. 97-104, 2016. 
[20] Z.-H. Ma, W. C. Chew, Y. M. Wu, and L. J. Jiang, "A new multilevel method for electrostatic problems through hierarchical loop basis," Computer Physics Communications, vol. 189, pp. 99-105, 2015.

[21] R. Jiwari, "A hybrid numerical scheme for the numerical solution of the Burgers' equation," Computer Physics Communications, vol. 188, pp. 59-67, 2015.

[22] D. K. Hoffman, G. W. Wei, D. S. Zhang, and D. J. Kouri, "Shannon-Gabor wavelet distributed approximating functional," Chemical Physics Letters, vol. 287, no. 1-2, pp. 119-124, 1998.

[23] K. Manoj and P. Sapna, "An efficient algorithm based on Haar wavelets for numerical simulation of Fokker-Planck equations with constants and variable coefficients," International Journal of Numerical Methods For Heat \& Fluid Flow, vol. 25, no. 1, pp. 41-56, 2015.

[24] R. C. Mittal, H. Kaur, and V. Mishra, "Haar wavelet-based numerical investigation of coupled viscous Burgers' equation," International Journal of Computer Mathematics, vol. 92, no. 8, pp. 1643-1659, 2015.

[25] S. Ul-Islam, I. Aziz, and A. S. Al-Fhaid, "An improved method based on Haar wavelets for numerical solution of nonlinear integral and integro-differential equations of first and higher orders," Journal of Computational and Applied Mathematics, vol. 260, pp. 449-469, 2014.

[26] P. K. Sahu and S. Saha Ray, "A new approach based on semiorthogonal B-spline wavelets for the numerical solutions of the system of nonlinear Fredholm integral equations of second kind," Computational and Applied Mathematics, vol. 33, no. 3, pp. 859-872, 2014.

[27] A. Tavakoli, F. Pourakbari, and H. Negarestani, "Adaptive multiple knot B-spline wavelets for solving saint-venant equations," International Journal of Wavelets Multiresolution and Information Processing, vol. 11, no. 6, 2013.

[28] P. K. Sahu and S. S. Ray, "Legendre wavelets operational method for the numerical solutions of nonlinear Volterra integro-differential equations system," Applied Mathematics and Computation, vol. 256, pp. 715-723, 2015.

[29] M. Tahami, A. A. Hemmat, and S. A. Yousefi, "Numerical solution of two-dimensional first kind Fredholm integral equations by using linear Legendre wavelet," International Journal of Wavelets Multiresolution and Information Processing, vol. 14, no. 1, 2016.

[30] L.-W. Liu, "Interval wavelet numerical method on fokkerplanck equations for nonlinear random system," Advances in Mathematical Physics, vol. 2013, Article ID 651357, 7 pages, 2013.

[31] H. yan, "Adaptive wavelet precise integration method for nonlinear black-scholes model based on variational iteration method," Abstract and Applied Analysis, vol. 2013, Article ID 735919, 6 pages, 2013.

[32] S. Mei and W. Gao, "Shannon-Cosine wavelet spectral method for solving fractional Fokker-Planck equations," International Journal of Wavelets, Multiresolution and Information Processing, vol. 16, no. 3, Article ID 1850021, 2018.

[33] S. Mei, X. Liu, and S. Mei, "Cell-filtering-based multi-scale Shannon-Cosine wavelet denoising method for locust slice image," International Journal of Wavelets, Multiresolution and Information Processing, vol. 17, no. 3, Article ID 1950035, 22 pages, 2019.

[34] A. Aldroubi, M. Unser, and A. Aldroubi, "Sampling procedures in function spaces and asymptotic equivalence with shannon's sampling theory," Numerical Functional Analysis and Optimization, vol. 15, no. 1-2, pp. 1-21, 1994.
[35] I. Kharitonenko, S. Xing Zhang, and S. Twelves, "A wavelet transform with point-symmetric extension at tile boundaries," IEEE Transactions on Image Processing, vol. 11, no. 12, pp. 1357-1364, 2002.

[36] S.-L. Mei and D. Zhu, "Interval shannon wavelet collocation method for fractional fokker-planck equation," Advances in Mathematical Physics, vol. 2013, Article ID 821820, 12 pages, 2013. 